Pacific Journal of Mathematics

WERSION OF THE HANKLL POTENTIL TRANSFOOM 


\section{INVERSION OF THE HANKEL POTENTIAL TRANSFORM}

Frank M. Cholewinski and Deborah Tepper Haimo

We consider the Hankel potential transform

$$
f(x)=\int_{0}^{\infty} \frac{t}{\left(x^{2}+t^{2}\right)^{\nu+1}} \phi(t) d \mu(t), \nu>0,
$$

where

$$
d \mu(x)=\frac{1}{2^{\nu-1 / 2} \Gamma(v+1 / 2)} x^{2 \nu} d x .
$$

This transform is intimately related to the Hankel transform. Indeed, its kernel is the Hankel transform

$$
\frac{t}{\left(x^{2}+t^{2}\right)^{\nu+1}}=\frac{\sqrt{\pi}}{2^{\nu+1 / 2} \Gamma(\nu+1)} \int_{0}^{\infty} \mathscr{J}(x y) e^{-t x} d \mu(y),
$$

where

$$
\mathscr{J}(z)=2^{\nu-1 / 2} \Gamma\left(\nu+\frac{1}{2}\right) z^{-\nu+1 / 2} J_{\nu-1 / 2}(z),
$$

$J_{\gamma}(z)$ being the ordinary Bessel function of order $\gamma$. Our object is to develop an inversion theory for $(1.1)$ and to exploit the relationship of (1.1) to the Hankel transform.

When $\nu=0$, some of our results reduce, modulo a constant, to those of D. V. Widder in [6], a paper on which the present work is closely based. In [7], Widder derived an inversion theory for the general transform

$$
f(x)=\int_{0}^{\infty} K\left(\frac{x}{t}\right) \frac{\phi(t)}{t} d t,
$$

which includes his result in [6] as a special case; however, the transform (1.1) for $\nu>0$ is not covered by that development.

2. Preliminaries. The differential operator $L_{x}$ which is to effect the desired inversion of (1.1) is defined as follows:

$$
L_{x}[f(x)]=\lim _{n \rightarrow \infty} L_{n, x}[f(x)],
$$

where, with $D$ denoting differentiation with respect to $x$,

$$
\begin{gathered}
L_{1, x}[f]=\frac{\sqrt{\pi} \Gamma(2 \nu+1)}{2^{5 / 2+\nu}[\Gamma(2+\nu / 2)]^{2} x^{1+2 \nu}} D x^{5+2 \nu} D \frac{1}{x} D[f] . \\
L_{n, x}[f]=\frac{(-1)^{n+1} \sqrt{\pi} \Gamma(2 \nu+1)}{2^{2 n+\nu+1 / 2}[\Gamma(n+\nu / 2+1)]^{2} x^{2 n+2 \nu-1}} D x^{4 n+2 \nu+1} \\
\times\left[\prod_{k=1}^{n-1} D \frac{1}{x^{4 k+2 \nu+1}} D x^{4 k+2 \nu+1}\right] D \frac{1}{x} D[f], n=2,3, \cdots
\end{gathered}
$$


the operations to be performed in order of increasing $k, k=1,2, \cdots$, $n-1$. It may readily be established that

$$
L_{n+1, x}[f]=\frac{-1}{4(n+\nu / 2+1)^{2}} \frac{1}{x^{2 n+2 \nu+1}} D x^{4 n+2 \nu+5} D \frac{1}{x^{2 n+2}} L_{n, x}[f] .
$$

Hence, an induction argument establishes the validity of

$$
L_{n, x}\left[\frac{1}{\left(x^{2}+t^{2}\right)^{\nu+1}}\right]=\frac{2^{\nu+1 / 2} \Gamma(\nu+1 / 2) \Gamma(2 n+\nu+2)}{[\Gamma(n+\nu / 2+1)]^{2}} \frac{x^{2 n+2} t^{2 n}}{\left(x^{2}+t^{2}\right)^{2 n+\nu+2}} .
$$

We note that an alternative form for the operator $L_{n, x}$ is given by

$$
L_{n, x}=-\frac{2^{\nu-1 / 2} \Gamma(\nu+1 / 2) \Gamma(n+\nu+1) n !}{[\Gamma(n+\nu / 2+1)]^{2}} \theta \prod_{k=1}^{n}\left(1-\frac{\theta}{2 k}\right)\left(1+\frac{\theta}{2 k+2 \nu}\right),
$$

where

$$
\theta=x D \text { and } \theta^{2}=x D(x D) .
$$

Since $\theta\left[x^{\alpha}\right]=\alpha x^{\alpha}$ and $\theta^{2}\left[x^{\alpha}\right]=\alpha^{2} x^{\alpha}$, we have, from (2.5), that

$$
\begin{aligned}
L_{n, x}\left[x^{\alpha}\right]= & -\frac{2^{\nu-1 / 2} \Gamma(\nu+1 / 2) \Gamma(n+\nu+1) n !}{[\Gamma(n+\nu / 2+1)]^{2}} \\
& \times \alpha \prod_{k=1}^{n}\left(1-\frac{\alpha}{2 k}\right)\left(1+\frac{\alpha}{2 k+2 \nu}\right) x^{\alpha},
\end{aligned}
$$

so that it is clear that the operator $L_{n, x}$ annihilates positive even powers of $x$. Letting $n \rightarrow \infty$ in (2.7), we find that

$$
L_{x}\left[x^{\alpha}\right]=-\left[\frac{\sqrt{\pi} \Gamma(2 \nu+1)}{2^{\nu+1 / 2} \Gamma(\nu+1+\alpha / 2) \Gamma(1-\alpha / 2)}\right] \alpha x^{\alpha} .
$$

See p. 5 of [1].

A function $\phi(x)$ will belong to $L$ for $x \in[0, R), 0<R \leqq \infty$, if

$$
\int_{0}^{R}|\phi(x)| d \mu(x)<\infty \text {. }
$$

3. Inversion. We establish our principal inversion formula for the transform (1.1).

THEOREM 3.1. Let $\phi(t)$ belong to $L$ for $t \in[0, R]$ for every positive $R$, and let the integral

$$
f(x)=\int_{0}^{\infty} \frac{t}{\left(x^{2}+t^{2}\right)^{\nu+1}} \dot{\phi}(t) d \mu(t)
$$

converge for some $x \neq 0$. Then 


$$
\lim _{n \rightarrow \infty} L_{n, x}[f(x)]=\dot{\phi}(x),
$$

at all points $x$ of the Lebesgue set for the function $\phi(x)$.

Proof. We seek to prove (3.2) for all $t$ for which

$$
\int_{t}^{x}|\dot{\phi}(u)-\dot{\phi}(t)| d \mu(u)=o(|x-t|),
$$

An appeal to Theorem 2c, p. 328, of [5] establishes that the integral (3.1) converges for all $x \neq 0$ and that differentiation under the integral sign is valid. Hence, using (2.4), we find that

$$
\begin{aligned}
L_{n, x}[f(x)] & =\frac{2^{\nu+1 / 2} \Gamma(\nu+1 / 2) \Gamma(2 n+\nu+2)}{[\Gamma(n+\nu / 2+1)]^{2}} \int_{0}^{\infty} \frac{x^{2 n+2} t^{2 n+1}}{\left(x^{2}+t^{2}\right)^{2 n+\nu+2}} \dot{\varphi}(t) d \mu(t) \\
& =\frac{2 \Gamma(2 n+\nu+2)}{[\Gamma(n+\nu / 2+1)]^{2}} \int_{0}^{\infty}\left(\frac{t}{1+t^{2}}\right)^{2 n+2} \frac{\phi(x t)}{t^{1-2 \nu}\left(1+t^{2}\right)^{\nu}} d t
\end{aligned}
$$

We may now apply the asymptotic estimate of Corollary 2b.2, p. 279, of [5] to

$$
\int_{0}^{1}\left(\frac{t}{1+t^{2}}\right)^{2 n+2} \frac{\phi(x t)}{t^{1-2 \nu}\left(1+t^{2}\right)^{\nu}} d t
$$

and that of Theorem $2 \mathrm{~b}, \mathrm{p} .278$, of [5] to

$$
\int_{1}^{\infty}\left(\frac{t}{1+t^{2}}\right)^{2 n+2} \frac{\phi(x t)}{t^{1-2 \nu}\left(1+t^{2}\right)^{\nu}} d t
$$

to obtain

$$
L_{n, x}[f(x)] \sim \frac{\Gamma(2 n+\nu+2) \sqrt{\pi}}{2^{2 n+\nu+1}[\Gamma(n+\nu / 2+1)]^{2} \vee \bar{n}} \dot{\varphi}(x),
$$

Using Stiring's formula, we find that

$$
\lim _{n \rightarrow \infty} L_{n, x}[f(x)]=\dot{\phi}(x),
$$

and the proof is complete.

Corollary 3.2. (3.2) holds for almost all $x, x \in(0, \infty)$.

COROLlary 3.3. (3.2) holds for all $x$ for which $\phi(x)$ is continuous.

Corollary 3.4. At all points $x$ in a neighborhood of which $\dot{\phi}(x)$ is of bounded variation, we have

$$
L_{x}[f(x)]=\frac{\phi\left(x^{+}\right)+\phi\left(x^{-}\right)}{2} .
$$


An additional result which follows by the method of Theorems $3 \mathrm{~b}$, $3 \mathrm{c}$ of [9], pp. 282-283, is the following.

CoROllaRy 3.5. Let $\phi(t)$ belong to $L$ for $t \in(\varepsilon, R)$ for every $\varepsilon, R$, $0<\varepsilon<R$, and let the integral

$$
f(x)=\int_{\varepsilon}^{R} \frac{t}{\left(x^{2}+t^{2}\right)^{\nu+1}} \dot{\phi}(t) d \mu(t)
$$

converge for some $x \neq 0$, and the integral

$$
\int_{0^{+}}^{1} \phi(x) x^{r} d x
$$

converge for some fixed $r$. Then

$$
\lim _{n \rightarrow \infty} L_{n, x}[f(x)]=\dot{\phi}(x)
$$

at all points $x$ of the Lebesgue set for the function $\phi(x)$.

As an example illustrating the theorem, consider the Hankel transform

$$
\int_{0}^{\infty} \frac{\mathscr{J}(t y)}{t} \frac{t}{\left(x^{2}+t^{2}\right)^{\nu+1}} d \mu(t)=\frac{\sqrt{\pi}}{2^{\nu+1 / 2} \Gamma(\nu+1)} \frac{e^{-x y}}{x} .
$$

Now

$$
\begin{aligned}
L_{n, x}\left[\frac{e^{-x y}}{x}\right]= & -\frac{2^{\nu-1 / 2} \Gamma(\nu+1 / 2) \Gamma(n+\nu+1) n !}{[\Gamma(n+\nu / 2+1)]^{2}} \sum_{j=0}^{\infty} \frac{(-1)^{j} y^{j}}{j !} \\
& \times(j-1) \prod_{k=1}^{n}\left(1-\frac{j-1}{2 k}\right)\left(1+\frac{j-1}{2 k+2 \nu}\right) x^{j-1}
\end{aligned}
$$

A straightforward computation gives

$$
L_{x}\left[\frac{e^{-x y}}{x}\right]=\frac{2^{\nu+1 / 2} \Gamma(\nu+1)}{\sqrt{\pi}} \frac{\mathscr{J}(x y)}{x},
$$

since taking the limit under the summation sign in (3.6) is valid due to the fact that the series of (3.6) is dominated by the series

$$
\sum_{j=0}^{\infty} \frac{1}{j !}(x y)^{j} e^{-/ 2|j-1|}
$$

which converges for all $x$. Hence the result predicted by the theorem is derived.

Other examples where the validity of the theorem may likewise be verified are 


$$
f(x)=\int_{0}^{\infty} \frac{\cos t}{t^{1+2 \nu}} \frac{t}{\left(x^{2}+t^{2}\right)^{\nu+1}} d \mu(t)=\frac{K_{\nu+1 / 2}(x)}{x^{\nu+1 / 2} \Gamma(2 \nu+1)}
$$

where

$$
L_{x}\left[x^{-\nu-1 / 2} K_{\nu+1 / 2}(x)\right]=\Gamma(2 \nu+1) x^{-2 \nu-1} \cos x ;
$$

and

$$
\begin{aligned}
f(x)= & \int_{0}^{\infty} t^{-2} J_{2 \nu}(t) \frac{t}{\left(x^{2}+t^{2}\right)^{\nu+1}} d \mu(t) \\
= & \frac{\Gamma(1-\nu)}{2^{\nu+2} \sqrt{\pi} \nu \Gamma(2 \nu+1)}{ }_{1} F_{2}\left(2 \nu ; \nu, 2 \nu+1 ; \frac{x^{2}}{4}\right) \\
& +\frac{\Gamma(\nu-1)}{2^{-\nu-7 / 2} \sqrt{\pi}\left(\nu^{2}-1\right) \Gamma(2 \nu)}{ }_{1} F_{2}\left(1+\nu ; 2+\nu, 2+\nu ; \frac{x^{2}}{4}\right),
\end{aligned}
$$

where

$$
L_{x}[f(x)]=x^{-2} J_{2 \nu}(x)
$$

4. Relation to the Hankel transform. The relation of the Hankel potential transform to the Hankel transform enables us to derive an inversion of the latter in terms of the operator $L_{t}$. We have the following result.

Theorem 4.1. If $\dot{\phi}(u)$ belongs to $L$ for $0 \leqq t<\infty$, and if

$$
\dot{\phi}^{\wedge}(x)=\int_{0}^{\infty} \mathscr{F}(x u) \dot{\phi}(u) d \mu(u)
$$

then, for almost a!l t,

$$
\dot{\phi}(t)=\frac{\sqrt{\pi t}}{2^{\nu+1 / 2} \Gamma(\nu+1)} L_{t}\left[\frac{R(t)}{t}\right], 0<t<\infty,
$$

where

$$
R(t)=\int_{0}^{\infty} e^{-t u} \dot{\phi}^{\wedge}(u) d \mu(u)
$$

Proof. An appeal to Fubini's theorem establishes that

$$
\begin{aligned}
R(t) & =\int_{0}^{\infty} e^{-u t} d \mu(u) \int_{0}^{\infty} \mathscr{J}(u y) \dot{\phi}(y) d \mu(y) \\
& =\int_{0}^{\infty} \phi(y) d \mu(y) \int_{0}^{\infty} e^{-u t} \mathscr{J}(u y) d d \mu(u) \\
& =\frac{2^{\nu+1 / 2} \Gamma(\nu+1)}{V \bar{\pi}} t \int_{0}^{\infty} \frac{1}{\left(y^{2}+t^{2}\right)^{\nu+1}} \dot{\phi}(y) d \mu(y),
\end{aligned}
$$

and the proof is complete on application of Theorem 3.1.

If in addition to the conditions of the theorem, we assume that 
$\phi^{\wedge}(x) \in L$ for $0 \leqq x<\infty$, we obtain the familiar inversion of the Hankel transform given in the following corollary.

COROLlary 4.2. If $\phi(u)$ belongs to $L$ for $0 \leqq u<\infty$, and if

$$
\dot{\phi}^{\wedge}(x)=\int_{0}^{\infty} \mathscr{J}(x u) \dot{\phi}(u) d \mu(u)
$$

with $\phi^{\wedge}(x) \in L$ for $0 \leqq x<\infty$, then

$$
\dot{\phi}(u)=\int_{0}^{\infty} \mathscr{J}(x u) \phi^{\wedge}(x) d \mu(x) .
$$

Proof. We have, with the notation of the theorem,

$$
L_{t}\left[\frac{R(t)}{t}\right]=\lim _{n \rightarrow \infty} L_{n, t} \int_{0}^{\infty} \frac{e^{-i y}}{t} \dot{\phi}^{\wedge}(y) d \mu(y) .
$$

Since $\dot{\phi}^{\wedge}(x) \in L$, the operator $L_{n, t}$ may be taken under the integral sign, and, by the argument used to establish (3.7), the limit in (4.7) may be applied to the integrand directly. Hence we have, on taking note of (3.7),

$$
L_{t}\left[\frac{R(t)}{t}\right]=\frac{2^{\nu+1 / 2} \Gamma(\nu+1)}{V \bar{\pi}} \frac{1}{t} \int_{0}^{\infty} \mathscr{J}(t y) \dot{\varphi}^{\wedge}(y) d \mu(y) .
$$

Substituting (4.8) in (4.2), we derive the desired inversion (4.6).

Since the Hankel transform of the Hankel potential transform is a Laplace transform, we may use the inversion algorithm of the latter to obtain another inversion formula for the Hankel potential transform, as in the following result.

THEOREM 4.3. Let $\phi(u)$ belong to $L$ for $0 \leqq u<\infty$, and let

$$
f(x)=\int_{0}^{\infty} \frac{t}{\left(t^{2}+x^{2}\right)^{\nu+1}} \dot{\phi}(t) d \mu(t) .
$$

Then, for almost all $t$,

$$
\phi(t)=\Gamma(2 \nu+1) t^{-2 \nu} \lim _{k \rightarrow \infty} \mathscr{L}_{k, t} f^{\wedge}(t),
$$

where

$$
\mathscr{L}_{k, t} f(t)=\frac{(-1)^{k}}{k !} f^{(k)}\left(\frac{k}{t}\right)\left(\frac{k}{t}\right)^{k+1}
$$

and

$$
f^{\wedge}(t)=\int_{0}^{\infty} \mathscr{J}(t u) f(u) d \mu(u)
$$


Proof. Since $\phi(u) \in L$, it follows that $f(x) \in L$ for $0<x<\infty$. Hence, clearly, $f^{\wedge}(t)$ exists, and by Fubini's theorem, we have

$$
\begin{aligned}
f^{\wedge}(z) & =\int_{0}^{\infty} \mathcal{J}(z u) d \mu(u) \int_{0}^{\infty} \frac{t}{\left(t^{2}+u^{2}\right)^{\nu+1}} \phi(t) d \mu(t) \\
& =\int_{0}^{\infty} \phi(t) d \mu(t) \int_{0}^{\infty} \frac{t \mathscr{J}(z u)}{\left(t^{2}+u^{2}\right)^{\nu+1}} d \mu(u) \\
& =\frac{\pi^{1 / 2}}{2^{\nu+1 / 2} \Gamma(\nu+1)} \int_{0}^{\infty} e^{-t z} \phi(t) d \mu(t) .
\end{aligned}
$$

But the right hand side is a Laplace transform, and by Theorem $6 \mathrm{a}$, p. 288 of [5], (4.10) follows.

The following example illustrates the theorem. Let

$$
f(x)=\int_{0}^{\infty} \frac{t}{\left(t^{2}+x^{2}\right)^{\nu+1}} e^{-t} d \mu(t) .
$$

Hence

$$
f^{\wedge}(t)=\frac{1}{(t+1)^{2 \nu+1}}
$$

and

$$
\begin{aligned}
\mathscr{L}_{k, t}\left[f^{\wedge}(t)\right]= & \frac{\Gamma(2 \nu+1+k)}{k ! \Gamma(2 \nu+1)}\left(\frac{k}{k+t}\right)^{k+1}\left(\frac{t}{k+t}\right)^{2 \nu} \\
& \sim \frac{t^{2 \nu}}{\Gamma(2 \nu+1)} e^{-t}, k \longrightarrow \infty
\end{aligned}
$$

We thus have

$$
\begin{aligned}
\Gamma(2 \nu+1) t^{-2 \nu} \lim _{k \rightarrow \infty} \mathscr{L}_{k, t}\left[f^{\wedge}(t)\right] & =e^{-t} \\
& =\phi(t)
\end{aligned}
$$

as predicted.

5. Inversion for a function having a power series expansion. If $f(x)$ represented by the transform (1.1) has an expansion in powers of $x$, the inversion of (3.2) becomes especially simple. Indeed, an elementary, practical algorithm may be illustrated by the following example:

$$
\int_{0}^{\infty} \frac{t}{\left(x^{2}+t^{2}\right)^{2}} \frac{1}{t\left(1+t^{2}\right)} \sqrt{\frac{2}{\pi}} t^{2} d t=\frac{\sqrt{\pi}}{2^{3 / 2}} \frac{1}{x(1+x)^{2}} .
$$

To invert this transform, we expand $1 / x(1+x)^{2}$ in powers of $x$, cancel the odd powers, change the alternate signs, and replace the absolute value of each coefficient by $2^{3 / 2} / \sqrt{\pi}$. Illustrating each of the steps in this case, we have, on expanding in a power series 


$$
\frac{1}{x}\left\{1-2 x+3 x^{2}-4 x^{3}+5 x^{4}-6 x^{5}+\cdots\right\} ;
$$

cancelling odd powers and changing alternate signs, we obtain

$$
\frac{1}{x}\left\{1-3 x^{2}+5 x^{4}-7 x^{6}+\cdots\right\}
$$

and, finally, replacing the absolute value of each coefficient by $2^{3 / 2} / \sqrt{\pi}$, we find

$$
\frac{2^{3 / 2}}{\sqrt{\pi}} \frac{1}{x}\left\{1-x^{2}+x^{4}-x^{6}+\cdots\right\}=\frac{2^{3 / 2}}{\sqrt{\pi}} \frac{1}{x\left(1+x^{2}\right)},
$$

so that the algorithm applied to $\left(\sqrt{\pi} / 2^{3 / 2}\right) / x\left(1+x^{2}\right)$ yields $1 / x\left(1+x^{2}\right)$ as desired.

The general result which describes this algorithm is given in the following.

THEOREM 5.1. Let

$$
f(x)=\int_{0}^{\infty} \frac{t}{\left(x^{2}+t^{2}\right)^{\nu+1}} \dot{\phi}(t) d \mu(t),
$$

the integral converging for $x \neq 0$, and let

$$
f(x)=\frac{1}{x^{\alpha}} \sum_{k=0}^{\infty} a_{k} x^{k}, 0<x<\rho .
$$

Then

$$
\begin{aligned}
\phi(x)= & \frac{2^{1 / 2-\nu} \Gamma(2 \nu+1)}{\sqrt{\pi}} x^{-\alpha} \\
& \times\left[\sin \frac{\pi \alpha}{2} \sum_{k=0}^{\infty}(-1)^{k} a_{2 k} \frac{\Gamma(k+1-\alpha / 2)}{\Gamma(k+\nu+1-\alpha / 2)} x^{2 k}\right. \\
& \left.-\cos \frac{\pi \alpha}{2} \sum_{k=0}^{\infty}(-1)^{k} a_{2 k+1} \frac{\Gamma(3 / 2-\alpha / 2+k)}{\Gamma(3 / 2-\alpha / 2+k+\nu)} x^{2 k+1}\right]
\end{aligned}
$$

valid in some interval $0<x<\rho e^{-\pi / 2}$.

Proof. Since termwise differentiation is valid, we have

$$
\begin{aligned}
L_{n, x}[f(x)]= & -\frac{2^{\nu-1 / 2} \Gamma(\nu+1 / 2) \Gamma(n+\nu+1) n !}{[\Gamma(n+\nu / 2+1)]^{2}} \sum_{k=1}^{\infty} a_{k}(k-\alpha) \\
& \times \prod_{j=1}^{n}\left(1-\frac{k-\alpha}{2 j}\right)\left(1+\frac{k-\alpha}{2 j+2 \nu}\right) x^{k-\alpha},
\end{aligned}
$$

or, if we take the limit as $n \rightarrow \infty$ under the summation sign, we find that 


$$
\begin{aligned}
L_{x}[f(x)]= & -2^{\nu-1 / 2} \Gamma\left(\nu+\frac{1}{2}\right) \sum_{k=0}^{\infty} a_{k} \\
& \times \frac{(k-\alpha) \Gamma(\nu+1)}{\Gamma(1+\nu+k / 2-\alpha / 2) \Gamma(1-k / 2-\alpha / 2)} x^{k-\alpha} \\
= & -2^{\nu-1 / 2} \Gamma\left(\nu+\frac{1}{2}\right) \Gamma(\nu+1) x^{-\alpha} \\
& \times\left\{\sum_{k=0}^{\infty} a_{2 k} \frac{(2 k-\alpha)}{\Gamma(1+\nu+k-\alpha / 2) \Gamma(1-k+\alpha / 2)} x^{2 k}\right. \\
& \left.+\sum_{k=0}^{\infty} a_{2 k+1} \frac{(2 k+1-\alpha)}{\Gamma(3 / 2-\alpha / 2+\nu+k) \Gamma(1 / 2+\alpha / 2-k)} x^{2 k+1}\right\} .
\end{aligned}
$$

The left hand side of (5.6) is equal to $\phi(x)$ by Theorem 3.1, and the right hand side reduces to that given in (5.4) by a simple computation.

The validity of taking the limit in (5.5) and obtaining (5.6) follows from the fact that

$$
\begin{aligned}
\left|L_{n, x}[f(x)]\right| \leqq & \frac{2^{\nu-1 / 2} \Gamma(\nu+1 / 2) \Gamma(n+\nu+1) n !}{[\Gamma(n+\nu / 2+1)]^{2}} \\
& \times \sum_{k=0}^{\infty}\left|a_{k}\right||k-\alpha| \prod_{j=1}^{\infty}\left(1+\frac{(k-\alpha)^{2}}{4 j^{2}}\right) x^{k-\alpha} \\
= & \frac{2^{\nu+1 / 2} \Gamma(\nu+1 / 2) \Gamma(n+\nu+1) n !}{\pi[\Gamma(n+\nu / 2+1)]^{2}} x^{-\alpha} \\
& \times \sum_{k=0}^{\infty}\left|a_{k}\right| \sin h \frac{\pi|k-\alpha|}{2} x^{k}
\end{aligned}
$$

so that the series of (5.6) converges uniformly in $n$ if

$$
\sum_{k=0}^{\infty}\left|a_{k}\right| x^{k} e^{\pi / 2|k-\alpha|}<\infty .
$$

But this is so for $0<x<\rho e^{-\pi / 2}$ and hence the proof is complete.

Note that if $\rho$ is replaced by $\infty$ in the hypothesis of the theorem, the conclusion holds in $(0, \infty)$. Further, if $\phi$ is known to be analytic at the start in $(0, \infty)$, then $(5.4)$ will determine $\phi$ completely by analytic continuation. For example, applying the theorem to (5.1), we note that $\alpha=1$ so that the second sum of (5.4) vanishes and we have

$$
\begin{aligned}
L_{x}\left[\frac{\sqrt{\pi}}{3^{3 / 2}} \frac{1}{x(1+x)^{2}}\right] & =\frac{\sqrt{\pi}}{2^{3 / 2}} L_{n} \sum_{k=0}^{\infty}(-1)^{k}(k+1) x^{k-1} \\
& =\frac{1}{x} \sum_{k=0}^{\infty}(-1)^{k} x^{2 k} \\
& =\frac{1}{x\left(1+x^{2}\right)} .
\end{aligned}
$$

Here $\phi(x)=1 / x\left(1+x^{2}\right)$ is analytic for $0<x<\infty$. Even though 
$\sum_{k=0}^{\infty}(-1)^{k} x^{2 k}$ is valid only for $|x|<1$, it nonetheless determines $\phi$ for all positive $x$ by analytic continuation.

For $f(x)$ having a power series expansion in terms of negative powers of $x$, we have the following companion result whose proof is analogous to that of the preceding theorem and hence will be omitted.

\section{THEOREM 5.2. Let}

$$
f(x)=\int_{0}^{\infty} \frac{t}{\left(x^{2}+t^{2}\right)^{\nu+1}} \phi(t) d \mu(t)
$$

the integral converging for $x \neq 0$, and let

$$
f(x)=\frac{1}{x^{\alpha}} \sum_{k=0}^{\infty} a_{k} x^{-k}, \rho<x<\infty .
$$

Then

$$
\begin{aligned}
\phi(x)= & \frac{2^{1 / 2-\nu} \Gamma(2 \nu+1)}{\sqrt{\pi}} x^{-\alpha} \\
& \times\left[\sin \pi\left(\frac{\alpha}{2}-\nu\right) \sum_{k=0}^{\infty}(-1)^{k} a_{2 k} \frac{\Gamma(k+\alpha / 2-\nu)}{\Gamma(k+\alpha / 2)} x^{-2 k}\right. \\
& \left.+\cos \pi\left(\frac{\alpha}{2}-\nu\right) \sum_{k=0}^{\infty}(-1)^{k} a_{2 k+1} \frac{\Gamma(1 / 2+\alpha / 2-\nu+k)}{\Gamma(1 / 2+\alpha / 2+k)} x^{-2 k-1}\right],
\end{aligned}
$$

valid in some interval $\rho e^{\pi / 2}<x<\infty$.

We may illustrate this theorem with example (5.1) by expanding $1 / x(1+x)^{2}$ in the series $x^{-3} \sum_{j=0}^{\infty}(-1)^{j}(j+1) x^{-j}$. In this case $\alpha=3$, and the second sum of (5.9) vanishes so that we have

$$
\phi(x)=x^{-3} \sum_{k=0}^{\infty}(-1)^{k} x^{-2 k}=\frac{1}{x\left(1+x^{2}\right)}
$$

as expected.

6. Hankel harmonic functions. Formula (1.3) shows that the kernel of the Hankel potential transform (1.1) is the Abel mean of the Bessel function associated with the Hankel transform. Thus, the kernel of (1.1) is the Poisson kernel, in its non-Hankel translated form, associated with the Hankel convolution of Hirschman and Delsarte [2]. The inversion formula for the Hankel potential transform can be applied to the Hankel harmonic functions associated with the HankelPoisson kernel. The Hankel harmonic functions also appear as a special case of the generalized axially symmetric potential theory of A. Weinstein [4].

Let $u(x, t)$ be of class $C^{2}$ in a region $R$ of $t \geqq 0$ such that, if $R$ 
contains a segment of the line $x=0$, then $u(x, t)=u(-x, t)$ in a neighborhood of such segments. The function $u(x, t)$ is said to be Hankel harmonic in $R$ if and only if

$$
\left(\Delta_{x}+\frac{\partial^{2}}{\partial t^{2}}\right) u(x, t)=0,
$$

for all $(x, t) \in R$, where

$$
\Delta_{x} f(x)=f^{\prime \prime}(x)+\frac{2 \nu}{x} f^{\prime}(x) .
$$

The Hankel-Poisson kernel is given by

$$
P(x, y, t)=\frac{\sqrt{\pi}}{2^{\nu+1 / 2} \Gamma(\nu+1)} \int_{0}^{\infty} e^{-t u} \mathscr{J}(x u) \mathscr{J}(y u) d \mu(u) .
$$

Note that, by (1.3), we have

$$
P(x, 0, t)=P(x, t)=\frac{t}{\left(x^{2}+t^{2}\right)^{2+1}} .
$$

Since $\Delta_{x} \mathscr{J}(x u)=-u^{2} \mathscr{J}(x u)$, it may readily be verified that $P(x, t)$ is a Hankel harmonic function in the half plane $t>0$.

We establish the following inversion for Hankel harmonic functions.

THEOREM 6.1. Let $u(x, t)$ be an even function of $x$ and a bounded Hankel harmonic function in the half plane $t \geqq 0$. Then

$$
L_{x}\left[\frac{u(0, x)}{x}\right]=\frac{2^{\nu+1 / 2} \Gamma(\nu+1)}{\sqrt{\pi}} \frac{u(x, 0)}{x}, \quad x \neq 0 .
$$

Proof. From [3], it follows that

$$
u(x, t)=\frac{2^{\nu+1 / 2} \Gamma(\nu+1)}{\sqrt{\pi}} \int_{0}^{\infty} P(x, y, t) u(y, 0) d \mu(y), t>0 .
$$

Therefore,

$$
u(0, t)=\frac{2^{\nu+1 / 2} \Gamma(\nu+1)}{\sqrt{\pi}} \int_{0}^{\infty} \frac{t}{\left(y^{2}+t^{2}\right)^{1+\nu}} u(y, 0) d \mu(y),
$$

or, equivalently,

$$
\frac{u(0, t)}{t}=\frac{2^{\nu+1 / 2} \Gamma(\nu+1)}{\sqrt{\pi}} \int_{0}^{\infty} \frac{y}{\left(y^{2}+t^{2}\right)^{1+\nu}} \frac{u(y, 0)}{y} d \mu(y),
$$

for all $t>0$. Applying the inversion formula, we obtain (6.4), and the proof is complete.

As an illustrative example, consider the function 


$$
u(x, t)=e^{-a t} \mathscr{J}(a x) \text {. }
$$

Then $u(x, t)$ is even in $x$ and is a bounded Hankel harmonic function for $t \geqq 0$. An appeal to (3.7) provides the verification of the inversion (6.4).

\section{REFERENCES}

1. A. Erdelyi et al Higher transcendental functions, vol. 1, McGraw-Hill, New York, 1958.

2. I. I. Hirschman, Jr., Variation diminishing Hankel transforms, J. Analyse Math. 8 (1960-61), 307-336.

3. B. Muckenhaupt and E. M. Stein, Classical expansions and their relation to conjugate harmonic functions, Trans. Amer. Math. Soc., 118 (1965), 17-92.

4. A. Weinstein, Generalized axially symmetric potential theory, Bull. Amer. Math. Soc., 59 (1953), 20-38.

5. D. V. Widder, The Laplace transform, Princeton Univ. Press. Princeton, New Jersey, 1941.

6. - A transform related to the Poisson integral for a half plane, Duke Math.

J. 33 (1966), 355-362.

7. Inversion of a convolution transform by use of series, J. Analyse Math.

19 (1968), 293-312.

Received November 2, 1970 and in revised form January 13, 1971. The research of the first author was supported by the National Science Foundation grant GP-23118 and that of the second by National Science Foundation grant 20536.

Clemson University

AND

University of MisSOURI-St. LOUIS 


\section{PACIFIC JOURNAL OF MATHEMATICS}

\section{EDITORS}

\author{
H. SAMElson \\ Stanford University \\ Stanford, California 94305 \\ C. R. HOBBY \\ University of Washington \\ Seattle, Washington 98105
}

J. DugundjI

Department of Mathematics University of Southern California Los Angeles, California 90007

RICHARD ARENS

University of California

Los Angeles, California 90024

\section{ASSOCIATE EDITORS}
E. F. BECKENBACH
B. H. NeUmanN
F. WOLE
K. YOSHIDA

\section{SUPPORTING INSTITUTIONS}

\author{
UNIVERSITY OF BRITISH COLUMBIA \\ CALIFORNIA INSTITUTE OF TECHNOLOGY \\ UNIVERSITY OF CALIFORNIA \\ MONTANA STATE UNIVERSITY \\ UNIVERSITY OF NEVADA \\ NEW MEXICO STATE UNIVERSITY \\ OREGON STATE UNIVERSITY \\ UNIVERSITY OF OREGON \\ OSAKA UNIVERSITY \\ UNIVERSITY OF SOUTHERN CALIFORNIA
}

\author{
STANFORD UNIVERSITY \\ UNIVERSITY OF TOKYO \\ UNIVERSITY OF UTAH \\ WASHINGTON STATE UNIVERSITY \\ UNIVERSITY OF WASHINGTON \\ * * * \\ AMERICAN MATHEMATICAL SOCIETY \\ CHEVRON RESEARCH CORPORATION \\ NAVAL WEAPONS CENTER
}

The Supporting Institutions listed above contribute to the cost of publication of this Journal, but they are not owners or publishers and have no responsibility for its content or policies.

Mathematical papers intended for publication in the Pacific Journal of Mathematics should be in typed form or offset-reproduced, (not dittoed), double spaced with large margins. Underline Greek letters in red, German in green, and script in blue. The first paragraph or two must be capable of being used separately as a synopsis of the entire paper. The editorial "we" must not be used in the synopsis, and items of the bibliography should not be cited there unless absolutely necessary, in which case they must be identified by author and Journal, rather than by item number. Manuscripts, in duplicate if possible, may be sent to any one of the four editors. Please classify according to the scheme of Math. Rev. Index to Vol. 39. All other communications to the editors should be addressed to the managing editor, Richard Arens, University of California, Los Angeles, California, 90024.

50 reprints are provided free for each article; additional copies may be obtained at cost in multiples of 50 .

The Pacific Journal of Mathematics is published monthly. Effective with Volume 16 the price per volume (3 numbers) is $\$ 8.00$; single issues, $\$ 3.00$. Special price for current issues to individual faculty members of supporting institutions and to individual members of the American Mathematical Society: $\$ 4.00$ per volume; single issues $\$ 1.50$. Back numbers are available.

Subscriptions, orders for back numbers, and changes of address should be sent to Pacific Journal of Mathematics, 103 Highland Boulevard, Berkeley, California, 94708.

PUBLISHED BY PACIFIC JOURNAL OF MATHEMATICS, A NON-PROFIT CORPORATION

Printed at Kokusai Bunken Insatsusha (International Academic Printing Co., Ltd.), 7-17, Fujimi 2-chome, Chiyoda-ku, Tokyo, Japan. 


\section{Pacific Journal of Mathematics}

\section{Vol. 37, No. $2 \quad$ February, 1971}

Charles Compton Alexander, Semi-developable spaces and quotient images of metric spaces .................................... 277

Ram Prakash Bambah and Alan C. Woods, On a problem of Danzer. . . . . . . . . 295

John A. Beekman and Ralph A. Kallman, Gaussian Markov expectations and related integral equations . ....................................

Frank Michael Cholewinski and Deborah Tepper Haimo, Inversion of the Hankel

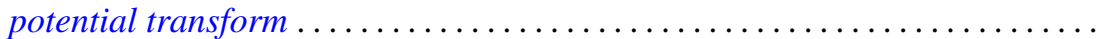

John H. E. Cohn, The diophantine equation

$$
Y(Y+1)(Y+2)(Y+3)=2 X(X+1)(X+2)(X+3) \ldots \ldots \ldots \ldots \ldots
$$

Philip C. Curtis, Jr. and Henrik Stetkaer, A factorization theorem for analytic

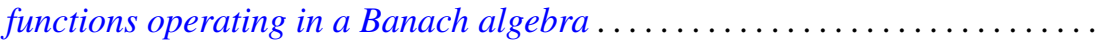

Doyle Otis Cutler and Paul F. Dubois, Generalized final rank for arbitrary limit ordinals

Keith A. Ekblaw, The functions of bounded index as a subspace of a space of entire functions

Dennis Michael Girard, The asymptotic behavior of norms of powers of

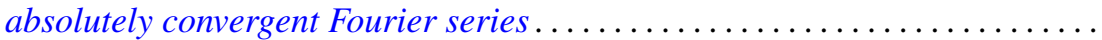

John Gregory, An approximation theory for elliptic quadratic forms on Hilbert spaces: Application to the eigenvalue problem for compact quadratic forms. 383

Paul C. Kainen, Universal coefficient theorems for generalized homology and stable cohomotopy.

Aldo Joram Lazar and James Ronald Retherford, Nuclear spaces, Schauder bases, and Choquet simplexes.

David Lowell Lovelady, Algebraic structure for a set of nonlinear integral operations

John McDonald, Compact convex sets with the equal support property . 429

Forrest Miller, Quasivector topologies

Marion Edward Moore and Arthur Steger, Some results on completability in commutative rings.

A. P. Morse, Taylor's theorem

Richard E. Phillips, Derek J. S. Robinson and James Edward Roseblade, Maximal subgroups and chief factors of certain generalized soluble groups.

Doron Ravdin, On extensions of homeomorphisms to homeomorphisms ...

John William Rosenthal, Relations not determining the structure of $\mathrm{L}$

Prem Lal Sharma, Proximity bases and subbases ........... .

Larry Smith, On ideals in $\Omega_{*}^{u}$. .

Warren R. Wogen, von Neumann algebras generated by operators similar to normal operators

R. Grant Woods, Co-absolutes of remainders of Stone-Čech 